PII: S0749-6419(97)00052-1

\title{
AN ANISOTROPIC ELASTIC-PLASTIC CONSTITUTIVE MODEL FOR SINGLE AND POLYCRYSTALLINE METALS. II-EXPERIMENTS AND PREDICTIONS CONCERNING THIN-WALLED TUBULAR OFHC COPPER*
}

\author{
A. S. Khan and P. Cheng ${ }^{\dagger}$ \\ Department of Mechanical Engineering, ECS 234, University of Maryland, Baltimore County, \\ Baltimore, MD 21250, U.S.A.
}

(Received in final revised form 26 July 1996)

\begin{abstract}
Predictions based on an anisotropic elastic-plastic constitutive model proposed in the first part of this paper are compared with the experimental stress and strain data on OHFC copper under first torsion to about $13 \%$ and partial unloading, and then tension-torsion to about $10 \%$ along eight different loading paths. This paper also describes the deformation and stress of the thin-walled tubular specimen under finite deformation, the numerical implementation of the model, and the detailed procedure for determining the material parameters in the model. Finally, the model is extended to a general representation of the multiple directors, and the elastic-viscoplastic extension of the constitutive model is considered. (C) 1998 Published by Elsevier Science Ltd. All rights reserved
\end{abstract}

Key words: B. Polycrystalline material.

\section{INTRODUCTION}

In the study of elastic-plastic behavior of polycrystalline metals, one of the main tasks is to describe the yield surface and its evolution with plastic deformation. At the beginning of plastic deformation, the yield surface is found (e.g. Taylor and Quinney, 1931) to be close to the von Mises criterion in stress space. After some plastic deformation, the yield surface changes in size, position and shape. If the plastic deformation is small, the change in shape is not as large as that in size and position (e.g. Naghdi et al., 1958; Phillips and Das, 1985), and thus the yield surface can be approximately expressed by a circle with a changed size and center, i.e. by a circle with isotropic and kinematic hardening. The evolution of isotropic and kinematic hardening with plastic deformation has been well studied and many models for it have been widely accepted. For example, the Prager (1949) and Ziegler (1959) linear model, the Armstrong and Frederick (1966) nonlinear model and the Dafalias and Popov (1976) two-surface model, have all been widely studied.

\footnotetext{
*Dedicated to the memory of Professor James F. Bell.

tVisiting scholar; permanent address: Institute of Mechanics, Chinese Academy of Sciences, Beijing 100080, People's Republic of China.
} 
The difficulty in describing the yield surface lies in the case of intermediate and finite plastic deformation. Stout et al.'s (1985) and Khan and Wang's (1993) experimental data on subsequent yield surfaces show that the shape change is as significant as the position change. If the assumption of the circular yield surface is maintained, the description is expected to depart from the experimental data (Khan and Parikh, 1986; Khan and Cheng, $1996 a$ ). If the yield surface is not supposed to be circular, other internal variables have to be added to the isotropic and kinematic hardening. The difficulty becomes more serious if the plastic deformation history is complicated and the yield surface is composed of several sections with different curvatures.

To describe the shape change of the yield surface under finite plastic deformation, Khan and Cheng (1996b) proposed a phenomenological model in the first part of this paper. In this model, there are many slip systems (material directors) which are randomly distributed in a material element. The critical resolved stresses in different directions form the yield surface. The relationship among the critical resolved stresses corresponds to the interaction of the material directors. If the interaction is approached by a polynomial of the angles among the material directors, the size, position and shape of the yield surface can be expressed in terms of the zero, first and higher degree, respectively. The evolution of the yield surface, including its shape, is accounted for by the change of the coefficients which change with plastic deformation. For the sake of convenience, hereafter this model will be called "multiple directors model".

This paper focuses on the correlation between the prediction by using this constitutive model and the experimental stress-strain data of OHFC copper under non-proportional loading as given by Khan and Wang (1993). The experiments were conducted on thinwalled tubular specimens. The experimental data includes the nominal stresses and the engineering strains under the following loading paths: first torsion to about $13 \%$ and partial unloading, and then torsion-tension at angles $0,15,30,45,60,75,90$ and $180^{\circ}$ to the unloading shear stress axis. To clarify the relation between the experimentally measured stresses and strains and those used in a general constitutive equation, Section II gives a description of the stress and deformation states of the thin-walled tubular specimen under finite deformation. The constitutive equation used in the description is specified in Section III. In Section IV, the general procedure of determining the parameters in the model is discussed and applied to the case of the experiments. Section $V$ provides the experimental data and the theoretical prediction of the model. This set of experimental data had been compared by Khan and Cheng (1996) with Chaboche's (1986) super-positional nonlinear model, the endochronic model (Valanis, 1971; Valanis and Lee, 1982; Watanabe and Atluri, 1986 and the two-surface model (Dafalias and Popov, 1976; Dafalias, 1983). To show the effect of the shape change of the yield surface on the stress-strain relation, Section $\mathrm{V}$ also gives some comparisons of the predictions of all the four models. Finally, the further development of the multiple directors model is discussed in Section VI; and some conclusions are given in Section VII.

\section{DEFORMATION AND STRESS OF THIN-WALLED TUBULAR SPECIMEN}

To describe a thin-walled tubular specimen, let $(R, \Theta, Z),\left(\mathbf{e}_{R}, \mathbf{e}_{\Theta}, \mathbf{e}_{Z}\right)$ and $(r, \theta, z)$, $\left(\mathbf{e}_{r}, \mathbf{e}_{\theta}, \mathbf{e}_{z}\right)$ be the cylindrical coordinates and the unit directions before and after deformation. Since the radial deformation of the specimen is generally much smaller than axial and angular deformations, the deformation of the specimen could be described as follows: 


$$
\begin{aligned}
& r=\alpha R \\
& \theta=\Theta+\omega Z \\
& z=\lambda Z
\end{aligned}
$$

where $\alpha$ and $\lambda$ are the radial and axial stretch ratios and $\omega$ is the angle of twist per unit undeformed length. From the description of deformation, deformation gradient $\mathbf{F}$ and velocity gradient $\mathbf{L}$ are found to be (e.g. Khan and Haung, 1995)

$$
\begin{aligned}
\mathbf{F}= & \alpha \mathbf{e}_{r} \otimes \mathbf{e}_{R}+\alpha \mathbf{e}_{\theta} \otimes \mathbf{e}_{\ominus}+\omega \alpha R \mathbf{e}_{\theta} \otimes \mathbf{e}_{Z}+\lambda \mathbf{e}_{z} \otimes \mathbf{e}_{Z} \\
\mathbf{L} & =\dot{\mathbf{F}} \cdot \mathbf{F}^{-1}=\frac{\dot{\alpha}}{\alpha} \mathbf{e}_{r} \otimes \mathbf{e}_{r}-\dot{\omega} Z \mathbf{e}_{r} \otimes \mathbf{e}_{\theta}+\dot{\omega} Z \mathbf{e}_{\theta} \otimes \mathbf{e}_{r} \\
& +\frac{\dot{\alpha}}{\alpha} \mathbf{e}_{\theta} \otimes \mathbf{e}_{\theta}+\dot{\phi} \mathbf{e}_{\theta} \otimes \mathbf{e}_{z}+\frac{\dot{\lambda}}{\lambda} \mathbf{e}_{z} \otimes \mathbf{e}_{z}
\end{aligned}
$$

where

$$
\dot{\phi}=\frac{\alpha R \dot{\omega}}{\lambda}
$$

It is noted that in obtaining eqn (3), the following geometrical relations have been used:

$$
\mathbf{e}_{r}=\dot{\theta} \quad \mathbf{e}_{\theta}=\dot{\omega} Z \mathbf{e}_{\theta}
$$

and

$$
\mathbf{e}_{\theta}=-\dot{\theta} \quad \mathbf{e}_{r}=-\dot{\omega} Z \mathbf{e}_{r}
$$

From eqn (3), the strain rate $\mathbf{D}$ and the material spin $\mathbf{W}$ are obtained.

$$
\begin{aligned}
\mathbf{D} & =\frac{1}{2}\left(\mathbf{L}+\mathbf{L}^{T}\right)=\frac{\dot{\alpha}}{\alpha} \mathbf{e}_{r} \otimes \mathbf{e}_{r}+\frac{\dot{\alpha}}{\alpha} \mathbf{e}_{\theta} \otimes \mathbf{e}_{\theta} \\
& +\frac{\dot{\phi}}{2} \mathbf{e}_{\theta} \otimes \mathbf{e}_{z}+\frac{\dot{\phi}}{2} \mathbf{e}_{z} \otimes \mathbf{e}_{\theta}+\frac{\dot{\lambda}}{\lambda} \mathbf{e}_{z} \otimes \mathbf{e}_{z}
\end{aligned}
$$

and

$$
\begin{aligned}
\mathbf{W} & =\frac{1}{2}\left(\mathbf{L}-\mathbf{L}^{T}\right)=-\dot{\omega} Z \mathbf{e}_{r} \otimes \mathbf{e}_{\theta}+\dot{\omega} Z \mathbf{e}_{\theta} \otimes \mathbf{e}_{r} \\
& +\frac{\dot{\phi}}{2} \mathbf{e}_{\theta} \otimes \mathbf{e}_{z}-\frac{\dot{\phi}}{2} \mathbf{e}_{z} \otimes \mathbf{e}_{\theta}
\end{aligned}
$$

Let $P$ and $M$ denote the tensile force and torsional moment acting on the specimen, the corresponding components of the Cauchy stress in the specimen will be

$$
\sigma_{z z}=\frac{P}{2 \pi t r}=\frac{p}{\alpha}
$$




$$
\sigma_{z \theta}=\frac{M}{2 \pi t r^{2}}=\frac{m}{\alpha^{2}}
$$

in which $t$ is the thickness of the specimen,

$$
m=\frac{M}{2 \pi t R^{2}}, \quad \text { and } \quad p=\frac{P}{2 \pi t R}
$$

are the nominal stress components.

As for other components of the Cauchy stress in the specimen, it is known that $\sigma_{r \theta}=\sigma_{r z}=0$, and $\sigma_{r r}=0$ since the specimen is a thin tube. But component $\sigma_{\theta \theta}$ cannot be known before the constitutive relation of the material is considered. As a result, the Cauchy stress in the specimen can be written as

$$
\boldsymbol{\sigma}=\sigma_{\theta \theta} \mathbf{e}_{\theta} \otimes \mathbf{e}_{\theta}+\frac{m}{\alpha^{2}} \mathbf{e}_{\theta} \otimes \mathbf{e}_{z}+\frac{m}{\alpha^{2}} \mathbf{e}_{z} \otimes \mathbf{e}_{\theta}+\frac{p}{\alpha} \mathbf{e}_{z} \otimes \mathbf{e}_{z}
$$

This stress and the above deformation produce the following material and Jaumann rates of the stress:

$$
\begin{aligned}
\dot{\boldsymbol{\sigma}} & =-Z \dot{\omega} \sigma_{\theta \theta} \mathbf{e}_{r} \otimes \mathbf{e}_{\theta}-\frac{m Z \dot{\omega}}{\alpha^{2}} \mathbf{e}_{r} \otimes \mathbf{e}_{z} \\
& -Z \dot{\omega} \sigma_{\theta \theta} \mathbf{e}_{\theta} \otimes \mathbf{e}_{r}+\dot{\sigma} \theta \theta \mathbf{e}_{\theta} \otimes \mathbf{e}_{\theta}+\frac{\alpha \dot{m}-2 m \dot{\alpha}}{\alpha^{3}} \mathbf{e}_{\theta} \otimes \mathbf{e}_{z} \\
& -\frac{m Z \dot{\omega}}{\alpha^{2}} \mathbf{e}_{z} \otimes \mathbf{e}_{r}+\frac{\alpha \dot{m}-2 m \dot{\alpha}}{\alpha^{3}} \mathbf{e}_{z} \otimes \mathbf{e}_{\theta}+\frac{\alpha \dot{p}-p \dot{\alpha}}{\alpha^{2}} \mathbf{e}_{z} \otimes \mathbf{e}_{z}
\end{aligned}
$$

and

$$
\begin{aligned}
\hat{\boldsymbol{\sigma}} & =\dot{\boldsymbol{\sigma}}-\mathbf{W} \cdot \boldsymbol{\sigma}+\boldsymbol{\sigma} \cdot \mathbf{W} \\
& =\left(\dot{\sigma}_{\theta \theta}-\frac{\dot{\phi} m}{\alpha^{2}}\right) \mathbf{e}_{\theta} \otimes \mathbf{e}_{\theta}+\left(\frac{\alpha \dot{m}-2 m \dot{\alpha}}{\alpha^{3}}+\left(\sigma_{\theta \theta}-\frac{p}{\alpha}\right) \frac{\dot{\phi}}{2}\right) \mathbf{e}_{\theta} \otimes \mathbf{e}_{z} \\
& +\left(\frac{\alpha \dot{m}-2 m \dot{\alpha}}{\alpha^{3}}+\left(\sigma_{\theta \theta}-\frac{p}{\alpha}\right) \frac{\dot{\phi}}{2}\right) \mathbf{e}_{z} \otimes \mathbf{e}_{\theta}+\left(\frac{\alpha \dot{p}-p \dot{\alpha}}{\alpha^{2}}-\frac{\dot{\phi} m}{\alpha^{2}}\right) \mathbf{e}_{z} \otimes \mathbf{e}_{z}
\end{aligned}
$$

The stress is related to the deformation through the constitutive response of the material. As usual, the constitutive relation between the Jaumann rate of Cauchy stress and strain rate follows this linear form:

$$
\hat{\boldsymbol{\sigma}}=\mathbf{C}: \mathbf{D}
$$

where $\mathbf{C}$ is the elastic-plastic modulus. Because the deformation considered here is only tension along and torsion around the axial direction of the tube, the components of the modulus with index $r \theta$ and $r z$ will be zero for initially isotropic materials. This makes the six component equations of eqn (15) reduce to four equations. By substituting eqns (7) and (14) into eqn (15), the four equations are found to be 


$$
\begin{aligned}
& A_{11} \frac{\dot{\lambda}}{\lambda}+A_{12} \dot{\phi}=\frac{\dot{p}}{\alpha} \\
& A_{21} \frac{\dot{\lambda}}{\lambda}+A_{22} \dot{\phi}=\frac{\dot{m}}{\alpha^{2}}
\end{aligned}
$$

and

$$
\begin{gathered}
\frac{\dot{\alpha}}{\alpha}=A \frac{\dot{\lambda}}{\lambda}+B \dot{\phi} \\
\dot{\sigma}_{\theta \theta}=C \frac{\dot{\lambda}}{\lambda}+D \dot{\phi}
\end{gathered}
$$

where

$$
\begin{gathered}
A=-\frac{C_{r r z z}}{C_{r r r}+C_{r r \theta \theta}} \\
B=-\frac{C_{r r \theta z}}{C_{r r r r}+C_{r r \theta \theta}} \\
C=C_{\theta \theta z z}+A C_{\theta \theta r r}+A C_{\theta \theta \theta \theta} \\
D=C_{\theta \theta \theta z}+\frac{m}{\alpha^{2}}+B C_{\theta \theta r r}+B C_{\theta \theta \theta \theta} \\
A_{11}=C_{z z z z}+A\left(C_{z z r r}+C_{z z \theta \theta}+\frac{p}{\alpha}\right) \\
A_{12}=C_{z z \theta z}+\frac{m}{\alpha^{2}}+B\left(C_{z z r r}+C_{z z \theta \theta}+\frac{p}{\alpha}\right) \\
A_{21}=C_{\theta z z z}+A\left(C_{\theta z r r}+C_{\theta z \theta \theta}+\frac{2 m}{\alpha^{2}}\right) \\
A_{22}=C_{\theta z \theta z}-\frac{1}{2}\left(\sigma_{\theta \theta}-\frac{p}{\alpha}\right)+B\left(C_{\theta z r r}+C_{\theta z \theta \theta}+\frac{2 m}{\alpha^{2}}\right)
\end{gathered}
$$

By solving eqns (16) and (17), deformation rates $\dot{\lambda}$ and $\dot{\omega}$ are related to loading rates $\dot{p}$ and $\dot{m}$ at any stage of loading or deformation in which deformation and stress, such as $\lambda, \alpha$ and $\sigma_{\theta \theta}$, are known. Subsequently, the deformation and stress rates, $\dot{\alpha}$ and $\dot{\sigma}_{\theta \theta}$, are found from eqns (18) and (19), and thus the complete state of deformation and stress rates are determined. In tension-torsion tests on the tube, only loadings $p$ and $m$ or deformation $\lambda$ and $\omega$ are controlled, while the stress $\sigma_{\theta \theta}$ and the deformation $\alpha$ are not known, all of which is reflected in this procedure for finding $\dot{\alpha}$ and $\dot{\sigma}_{\theta \theta}$. With the relation between the 
stress and deformation rates known, the relation between the total stress and deformation is established by integration step by step.

\section{IMPLEMENTATION OF THE CONSTITUTIVE MODEL}

In the first part of this paper, polycrystals are supposed to be associated with a large number of randomly distributed slip systems. For each slip system, say, the $i$ th slip system, let $\tau^{i}, r^{i}, x^{i}$ and $r_{0}$ denote the resolved shear stress, isotropic and kinematic hardening and the initial shear yield stress, respectively, i.e.

$$
\left|\tau^{i}-x^{i}\right| \leq r^{i}+r_{0}
$$

then the evolution equations of the isotropic and kinematic hardening with plastic deformation are assumed to be

$$
\dot{x}^{i}=\sum_{j}^{N} b_{x} \dot{\gamma}^{j}\left(X_{0} \delta^{i j}+X_{1} \mathbf{P}^{i}: \mathbf{P}^{j}+X_{3}\left(\mathbf{P}^{i}: \mathbf{P}^{j}\right)^{3}-\operatorname{sign}\left(\dot{\gamma}^{j}\right) x^{i}\right)
$$

and

$$
\dot{r}^{i}=\sum_{j}^{N} b_{r}\left|\dot{\gamma}^{j}\right|\left(R_{0}+R_{2}\left(\mathbf{P}^{i}: \mathbf{P}^{j}\right)^{2}-r^{i}\right)
$$

where $b_{x}, X_{1}, X_{3}, b_{r}, R_{0}, R_{2}$ and $X_{0}$ are material parameters, $N$ is the number of activated slip systems, $\dot{\gamma}^{i}$ is the slip rate and $\mathbf{P}^{i}$ is defined by the slip direction $\mathbf{m}^{i}$ and the slip plane $n^{i}$ such that

$$
\mathbf{P}^{i}=\frac{1}{2}\left(\mathbf{m}^{i} \otimes \mathbf{n}^{i}+\mathbf{n}^{i} \otimes \mathbf{m}^{i}\right)
$$

In other words, if the plastic behavior is described by

$$
\tau_{c}^{i}=\sum_{j}^{N} h^{i j} \dot{\gamma}^{j}
$$

then the work hardening $h^{i j}$ is

$$
\begin{aligned}
h^{i j} & =b_{x}\left(X_{0} \delta_{i j}+X_{1} \mathbf{P}^{i}: \mathbf{P}^{j}+X_{3}\left(\mathbf{P}^{i}: \mathbf{P}^{j}\right)^{3}-\operatorname{sign}\left(\dot{\gamma}^{j}\right) x^{i}\right) \\
& +\operatorname{sign}\left(\tau^{i}-x^{i}\right) b_{r} \operatorname{sign}\left(\dot{\gamma}^{j}\right)\left(R_{0}+R_{2}\left(\mathbf{P}^{i}: \mathbf{P}^{j}\right)^{2}-r^{i}\right)
\end{aligned}
$$

Written in the form of eqn (15), this model, an analogue to the plasticity theory of single crystals (e.g. Asaro, 1983), can be found to be

$$
\mathbf{C}=\mathbf{C}^{e}-\sum_{i} \sum_{j}\left(g^{i j}\right)^{-1} \lambda^{i} \otimes \lambda^{j}
$$


where

$$
g^{i j}=h^{i j}+\lambda^{i}: \mathbf{P}^{j}
$$

and

$$
\lambda^{i}=\mathbf{P}^{i}: \mathbf{C}^{e}-\boldsymbol{\sigma} \cdot \mathbf{Q}^{i}+\mathbf{Q}^{i} ; \boldsymbol{\sigma}
$$

and $\mathbf{C}^{\mathrm{e}}$ is the elastic modulus. If Hooke's law is assumed for the elastic behavior,

$$
\mathbf{C}^{e}=2 G \mathbf{I}_{4}+\frac{2 G v}{1-2 v} \mathbf{I} \otimes \mathbf{I}
$$

where $G$ is the shear modulus, $v$ is Poisson's ratio, $I$ and $\mathbf{I}_{4}$ are the unit second and fourth order tensors.

It is noted that the following relations have been applied in deriving the elastic-plastic modulus $\mathbf{C}$ :

$$
\begin{gathered}
\mathbf{L}=\mathbf{L}^{e}+\mathbf{L}^{p}, \quad \mathbf{L}^{p}=\sum_{i=1}^{N} \dot{\gamma}^{i} \mathbf{m}^{i} \otimes \mathbf{n}^{i} \\
\mathbf{m}^{i}=\mathbf{L}^{e} \cdot \mathbf{m}^{i}, \quad \dot{\mathbf{n}}^{i}=-\mathbf{n}^{i} \cdot \mathbf{L}^{e}
\end{gathered}
$$

and

$$
\dot{\gamma}^{i}=\sum_{j=1}^{N}\left(g^{i j}\right)^{-1} \lambda^{j}: \mathbf{L}
$$

Therefore, the numerical implementation of the model for the deformation of the specimen is straightforward. The deformation rates $\dot{\lambda}, \dot{\alpha}$ and $\dot{\phi}$ are found for a set of loading rates $\dot{p}$ and $\dot{m}$ from the combination of this model and the general relations of the tubular specimen given in the preceeding section. Subsequently, $\mathbf{L}, \dot{\gamma}^{i}, \mathbf{L}^{p}, \mathbf{L}^{e}, \dot{\mathbf{m}}^{i}$ and $\dot{\mathbf{n}}^{i}$ are determined one after another. When all the variables of the deformation and stress are changed to up-to-date values and the computation is repeated, this model predictions are obtained.

\section{DETERMINATION OF THE MATERIAL PARAMETERS}

In addition to two elastic constants, $G$ and $\nu$, eight material parameters are included in the multiple directors model for the description of anisotropic plastic behavior. Naturally, determining these material parameters begins with the understanding of their physical interpretation. For instance, the initial yield shear stress $r_{0}$ could be determined by a uniaxial tension test or simple shear test.

During the plastic deformation of polycrystals, it is known that most of plastic deformation corresponds to the activation of multiple slip systems. Therefore, it is understandable from eqn (29) that the parameter $X_{0}$ representing the independent hardening has much less contribution in value of the kinematic hardening $\dot{x}^{i}$ than $X_{1}$ and $X_{3}$, if they 
are of the same order. As a result, the value of $X_{0}$ is not significant in the model. However, the existence of $X_{0}$ plays a crucial role in providing the nonsingular $g^{i j}$ in eqn (34). If $X_{0}=0, g^{i j}$ is approximately $2 G \mathbf{P}^{i}: \mathbf{P}^{j}$ from eqns (34)-(37), and thus it becomes singular when the number of activated slip systems is more than five. Combining the two aspects, we suggest that $X_{0}=1$.

From eqns (31) and (39), it is found that

$$
\dot{\mathbf{P}}^{i}=\mathbf{D}^{e} \cdot \mathbf{Q}^{i}-\mathbf{Q}^{i} \cdot \mathbf{D}^{e}+\mathbf{W}^{e} \cdot \mathbf{P}^{i}-\mathbf{P}^{i} \cdot \mathbf{W}^{e}
$$

where $\mathbf{D}^{e}$ and $\mathbf{W}^{e}$ are, respectively, elastic strain rate and spin defined by the symmetrical and anti-symmetrical parts of $\mathbf{L}^{e}$, and that

$$
\left(\mathbf{P}^{i}: \mathbf{P}^{j}\right) \cdot=2 \mathbf{D}^{e}:\left(\mathbf{Q}^{i} \cdot \mathbf{P}^{j}+\mathbf{P}^{i} \cdot \mathbf{Q}^{j}\right)
$$

That is, even though the slip system $\mathbf{P}^{i}$ changes with elastic deformation and rotation, the angle $\mathbf{P}^{i}: \mathbf{P}^{j}$ between the $i$ th and $j$ th slip systems depends only on elastic deformation. Since elastic deformation is usually much smaller than plastic deformation in polycrystalline metals, elastic deformation could be ignored in the study of plastic behavior without significant error. With this consideration, $\mathbf{P}^{i}: \mathbf{P}^{j}$ could be taken to be constant during any deformation history.

With constant $\mathbf{P}^{i}: \mathbf{P}^{j}$, the sums of $X_{0} \delta^{i j}+X_{1} \mathbf{P}^{i}: \mathbf{P}^{j}+X_{3}\left(\mathbf{P}^{i}: \mathbf{P}^{j}\right)^{3}$ and $R_{0}+R_{2}\left(\mathbf{P}^{i}: \mathbf{P}^{j}\right)^{2}$ in eqns (29) and (30) are constants during deformation. From eqn (30), all activated slip systems contribute to the isotropic hardening of a slip system; the contribution of each activated slip system is proportional to the difference between the constant $R_{0}+R_{2}\left(\mathbf{P}^{i}: \mathbf{P}^{j}\right)^{2}$ and the isotropic hardening itself; when the $j$ th slip system is continuously activated, the isotropic hardening of the $i$ th slip system will continuously increase until it reaches the constant $R_{0}+R_{2}\left(\mathbf{P}^{i}: \mathbf{P}^{j}\right)^{2}$. Thus, the constant $R_{0}+R_{2}\left(\mathbf{P}^{i}: \mathbf{P}^{j}\right)^{2}$ is the saturated value of the cross isotropic hardening between the $i$ th and $j$ th slip systems, and consequently it is expected that $b_{r}>0$ and $R_{0}+R_{2}\left(\mathbf{P}^{i}: \mathbf{P}^{j}\right)^{2}>0$. Further, when $\mathbf{P}^{i}$ is the same as $\mathbf{P}^{j}, \mathbf{P}^{i}: \mathbf{P}^{j}=\frac{1}{2}$, and $R_{0}+R_{2}\left(\mathbf{P}^{i}: \mathbf{P}^{j}\right)^{2}=R_{0}+\frac{1}{4} R_{2}$. The latter represents the self hardening effect; when $\mathbf{P}^{i}$ is perpendicular to $\mathbf{P}^{j}, \mathbf{P}^{i}: \mathbf{P}^{j}=0$, and $R_{0}+R_{2}\left(\mathbf{P}^{i}: \mathbf{P}^{j}\right)^{2}=R_{0}$ represents the maximum cross hardening effect. Thus, $R_{2}$ is the maximum difference between self hardening and cross hardening, and $R_{0}$ is the phenomenological isotropic hardening. In light of the experimental result that a yield surface has the smallest size in the loading direction, the self hardening is expected to be smaller than the cross hardening. That is, $R_{2}<0$ and $R_{0}>-R_{2}$.

The similar analysis can be applied to eqn (29) to obtain the physical interpretation of its parameters. With the continuously activated $j$ th slip system, the kinematic hardening of the $i$ th slip system, $x^{i}$, keeps increasing until it reaches its saturated value $X_{0} \delta^{i j}+X_{1} \mathbf{P}^{i}: \mathbf{P}^{j}+X_{3}\left(\mathbf{P}^{i}: \mathbf{P}^{j}\right)^{3}$. In the saturated value, $X_{1}$ represents the phenomenological kinematic hardening. Thus, $b_{x}>0$ and $X_{1}>0$. As for $X_{3}$, since the yield surface tends to be sharper in loading direction and to be flatter in the opposite direction, $X_{3}$ should be positive. Moreover, compared to the isotropic hardening which is independent of the slip direction of an activated slip system, the kinematic hardening behaves differently whenever an activated slip system changes from a forward slip to a backward slip. This fact is expressed by the factor $\operatorname{sign}\left(\dot{\gamma}^{j}\right)$. When the backward slip continuously increases, $x^{i}$ decreases until it reaches the saturated value 
$-X_{0} \delta^{i j}-X_{1} \mathbf{P}^{i}: \mathbf{P}^{j}-X_{3}\left(\mathbf{P}^{i}: \mathbf{P}^{j}\right)^{3}$. Especially at the beginning of a backward slip, the hardening modulus $X_{0} \delta^{i j}+X_{1} \mathbf{P}^{i}: \mathbf{P}^{j}+X_{3}\left(\mathbf{P}^{i}: \mathbf{P}^{j}\right)^{3}+x^{i}$ is greater than the hardening modulus $X_{0} \delta^{i j}+X_{1} \mathbf{P}^{i}: \mathbf{P}^{j}+X_{3}\left(\mathbf{P}^{i}: \mathbf{P}^{j}\right)^{3}-x^{i}$ for the continuous forward slip.

It is worthwhile to point out that eqns (29) and (30) describe the evolution of the yield surface, and do not represent the relation between the yield surface and the slips and directions of slip systems. This method of describing hardening in a form of differential equation with respect to time is the same as in, e.g. Armstrong and Frederick's (1966) model for (phenomenological) kinematic hardening. This method is based on the experimental observation that a yield surface or hardening is dependent on plastic deformation history. Therefore, eqns (29) and (30) are, in general, unintegrable into direct relations between hardening and slips and directions. In some elastic-plastic constitutive models, e.g. Chaboche's (1986) nonlinear super-positional model and Dafalias and Popov's (1976) two surface model, the evolution of hardening with plastic deformation can be integrated in some specific cases, such as simple tension or simple torsion under infinitesimal deformation. But this model does not have this feature, because the evolution equations, eqns (29) and (30), specify the relation between hardening and slips and directions, instead of the hardening and phenomenological plastic deformation.

To specifically determine the material parameters for a given material, it is preferred to have an experimental yield surface as well as experimental stress-strain data on simple tension or torsion. From one dimensional experimental stress-strain data, the elastic shear modulus and the initial yield stress can be determined, if the elastic deformation is assumed to be isotropic. From the experimental yield surface, the saturated values of the hardening, $R_{0}, R_{2}$ and $X_{1}, X_{3}$ can be estimated. This provides a starting point to numerically determine these parameters and the shape-controlled parameters $b_{r}$ and $b_{x}$ through implementing the model. The detailed procedure of estimating these parameters is given in the following determination of the parameters for the fully annealed OHFC copper.

Figure 1 shows the loading paths of the tubular copper specimen. The specimen was first twisted along path $O \rightarrow C$ to $13 \%$ and underwent some unloading along $C \rightarrow U$, and reloaded with combined stretching and twisting at angles of $0,15,30,45,60,75,90$ and $180^{\circ}$ to the shear stress axis.

In terms of engineering stress and strain, the experimental data of simple shear deformation, the solid lines, are given in Fig. 2, in which curve OC is the first stage torsion, curve $\mathrm{CU}$ is the unloading before the nonproportional loading, curve UF is the reversal loading which corresponds to the loading path of $0^{\circ}$ in Fig. 1, and curve UCD is the reloading corresponding to the loading path of $180^{\circ}$. By using $0.2 \%$ offset strain as the yield criterion, the initial yield point and the reversal yield point are labelled by point $\mathrm{A}$ and point $\mathrm{E}$ in Fig. 2. From these data, the initial shear yield stress $r_{0}$ is found to be $1.732 \mathrm{ksi}$, and the elastic shear modulus $G$, is taken to be the average slope of OA and CE. The Poisson's ratio is assumed to be $1 / 3$.

Also from Fig. 2, the stress-strain data at the loading point $\mathrm{C}$ and the reloading point $\mathrm{E}$ can be found. At point $\mathrm{C}$, the stress and strain are $12.2 \mathrm{ksi}$ and $13.4 \%$. At point $\mathrm{E}$, the stress and strain are $-8.30 \mathrm{ksi}$ and $12.8 \%$. These data show that when plastically deformed to about $13 \%$, the material has isotropic hardening of $(12.2+8.30) / 2-1.732=8.518(\mathrm{ksi})$ and kinematic hardening of $(12.2-8.3) / 2=1.95(\mathrm{ksi})$. In addition, the loading slope around point $\mathrm{C}$ is much smaller than the reloading slope around point $\mathrm{E}$. This phenomenon suggests that the kinematic hardening has developed to a significant extent when plastic deformation reaches to $13 \%$. In other words, it would be a good approximation of the 


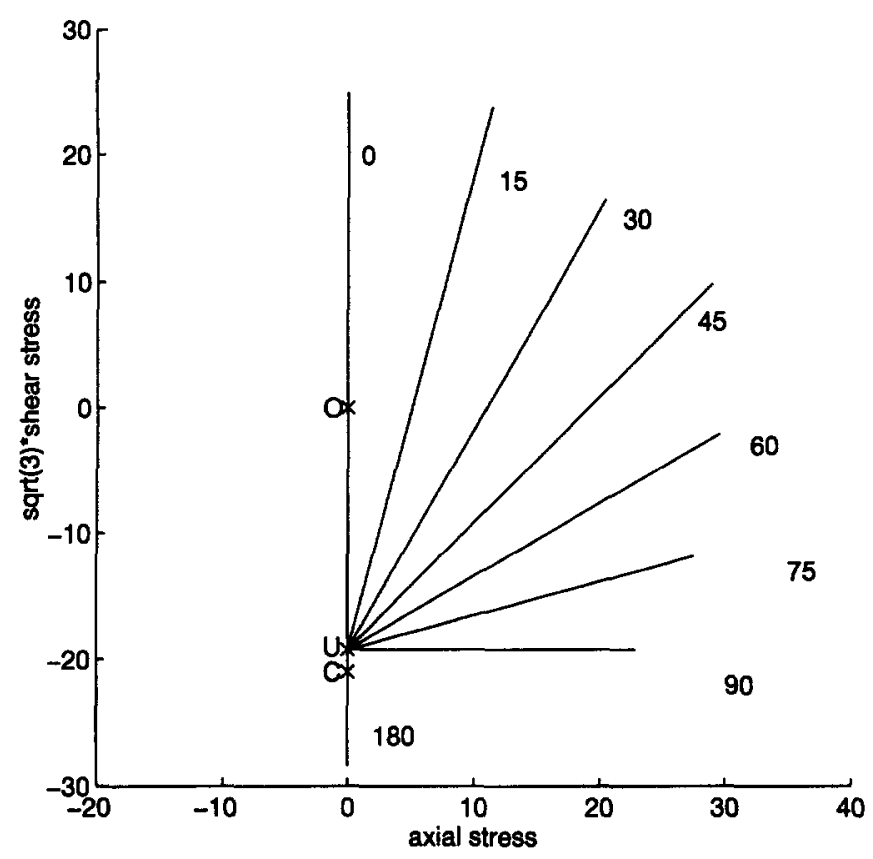

Fig. 1. Loading paths in the $\sigma-\sqrt{3} \tau$ space.

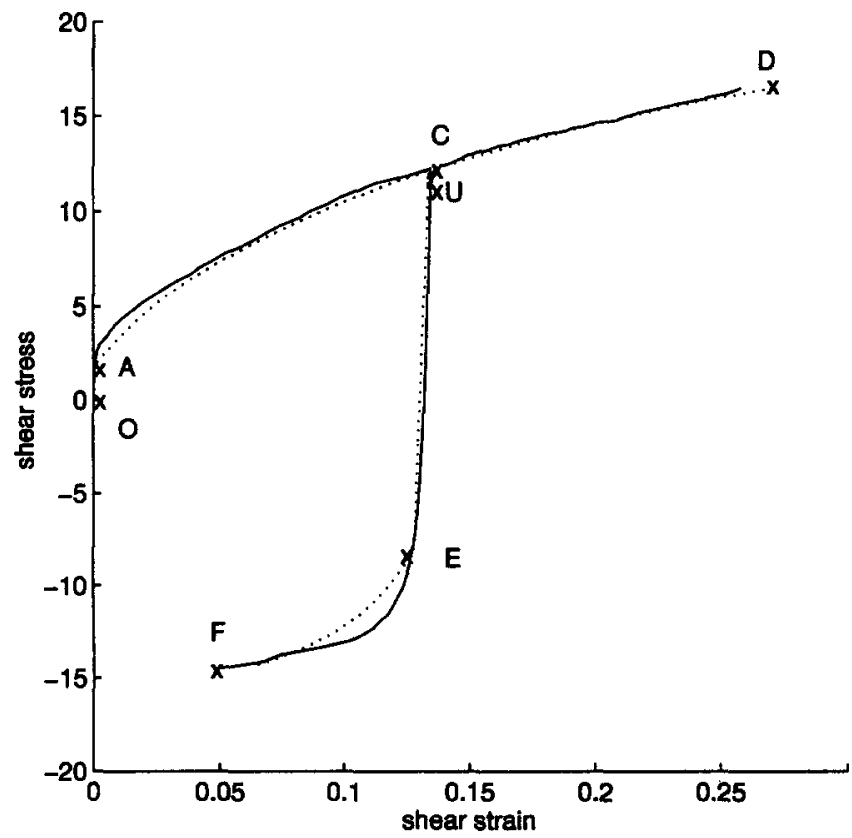

Fig. 2. Comparison of shear stress-strain relation under torsion deformation. __Experiments; …....prediction of the model. 
kinematic hardening if the saturated value of the kinematic hardening is taken to be a little larger than $1.95 \mathrm{ksi}$, say $2 \mathrm{ksi}$. Accordingly, since the kinematic hardening given by eqn (29) is approximately an exponential function of plastic deformation, then the shapecontrolled parameter $b_{x}$ should be a value, say 100 , such that it makes the kinematic hardening value almost equal to its saturated value when plastic deformation is about $13 \%$.

With these estimated parameters for kinematic hardening, the parameters for isotropic hardening can be further estimated. At point D in Fig. 2, the stress and strain are $16.7 \mathrm{ksi}$ and $26.9 \%$. By subtracting the estimated kinematic hardening and the initial yield stress from the stress, the isotropic hardening at this point is about $16.7-1.732-2=12.968(\mathrm{ksi})$. With the two isotropic hardening values and if the isotropic hardening given by eqn (30) is approximated by an exponential function, then the saturated value and the shape-controlled parameter $b_{r}$ for the isotropic hardening to be $17.82 \mathrm{ksi}$ and 5 .

The above estimated values, 17.82 and $2 \mathrm{ksi}$, of the saturated isotropic and kinematic hardening are based on the experimental data under simple shear deformation. They are the approximation of the terms $R_{0}+R_{2}\left(\mathbf{P}^{i}: \mathbf{P}^{j}\right)^{2}$ and $X_{1} \mathbf{P}^{i}: \mathbf{P}^{j}+X_{3}\left(\mathbf{P}^{i}: \mathbf{P}^{j}\right)^{3}$ in eqns (30) and (29), or in other words, $R_{0}+4 R_{2}$ and $2 X_{1}+8 X_{3}$ are approximately 17.82 and $1.95 \mathrm{ksi}$, since $-1 / 2 \leq \mathbf{P}^{i}: \mathbf{P}^{j} \leq 1 / 2$. In order to specifically estimate $R_{0}, R_{2}, X_{1}$ and $X_{3}$, the experimental yield surface at point $U$ in Fig. 1 is given by Fig. 3(a) and (b). Figure 3(b) is a representation of Fig. 3(a) in which $o$ is the angle between a direction originated at the stress point $(0,0)$ and the unloading direction or the negative shear stress axis. The isotropic and kinematic hardening is calculated by considering the yield surface in Fig. 3(a) to be symmetrical to the shear stress axis and by measuring the length minus the initial yield stress and the center in every direction. In Fig. 3(b), the isotropic hardening appears to be a quadratic function of $\cos o$ as shown by the dashed line with a maximum value of $10.35 \mathrm{ksi}$ and a minimum value of $8.518 \mathrm{ksi}$. If this ratio of $10.35 / 8.518=1.27$ is supposed to hold for $R_{0}$ to $R_{0}+4 R_{2}$, then $R_{0}=1.27\left(R_{0}+4 R_{2}\right)=1.27 \times 17.85=22.67(\mathrm{ksi})$ and $R_{2}=(17.85-22.67) / 4=-1.21$ (ksi). Moreover, Fig. 3(b) shows that the kinematic hardening is approximately a pure cubic function of $\cos o$ as shown by the dashed line. That suggests that $X_{1}$ could be supposed to be zero and $X_{3}$ to be the saturated value of $8 \times 2=16 \mathrm{ksi}$.

Starting with these estimated values, the parameters for the OHFC copper can be finally determined, with the goal that the parameters make the numerical prediction of the model as close to the whole set of experimental stress-strain data as possible. The final values of the parameters are found to be $R_{0}=23 \mathrm{ksi}, R_{2}=-1.5 \mathrm{ksi}, b_{r}=5, X_{1}=0$, $X_{3}=16 \mathrm{ksi}$ and $b_{x}=100$. Obviously, the elastic parameters and the initial yield stress do not need to be put into the numerical iterating process and should keep their values as obtained from the simple shear stress-strain data.

\section{EXPERIMENTAL DATA AND PREDICTION OF THE MODEL}

Figures 2. and 3(a) show that the model's determined material constants give a prediction that agrees well with the experimental data on the simple shear deformation, including the loading, unloading and reversal loading, and the yield surface after the first stage loading. The model is especially successful at predicting that a yield surface changes not only in its size and position, but also its shape. 

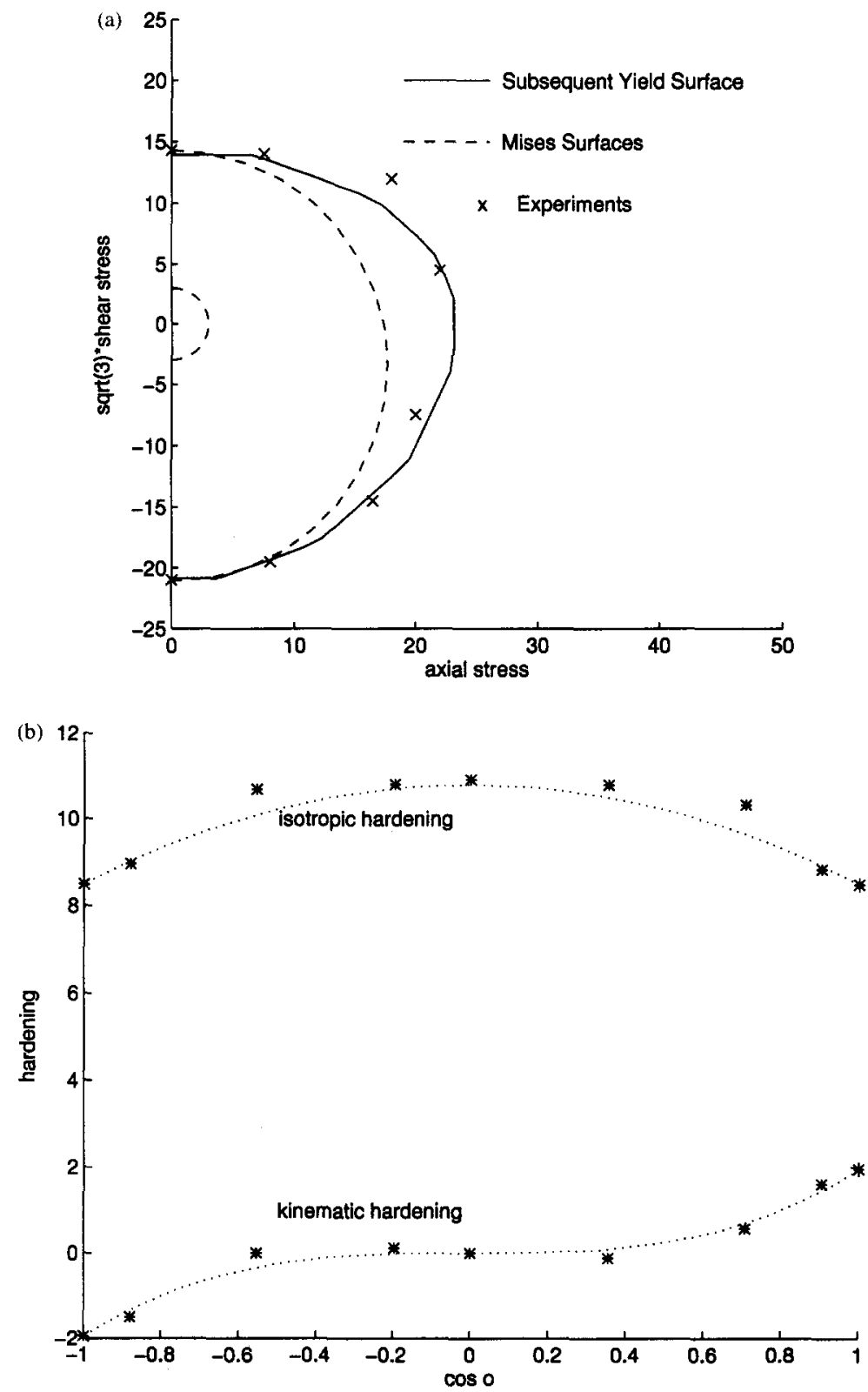

Fig. 3. Subsequent yield surface after torsional deformation: (a) experimental data and the prediction of the model; (b) experimental hardening versus the directions to the loading.

Figure 4 gives the experimental data and the prediction of the model concerning the axial and shear strains under the nonproportional loading paths of $15,30,45,60,75$ and $90^{\circ}$ in Fig. 1. For the sake of convenience, the strains are taken to be zero at the beginning of the additional loading, i.e. at $U$. This result shows that the model can predict the direction of plastic strain rate very well. In the model, all the framework follows the 


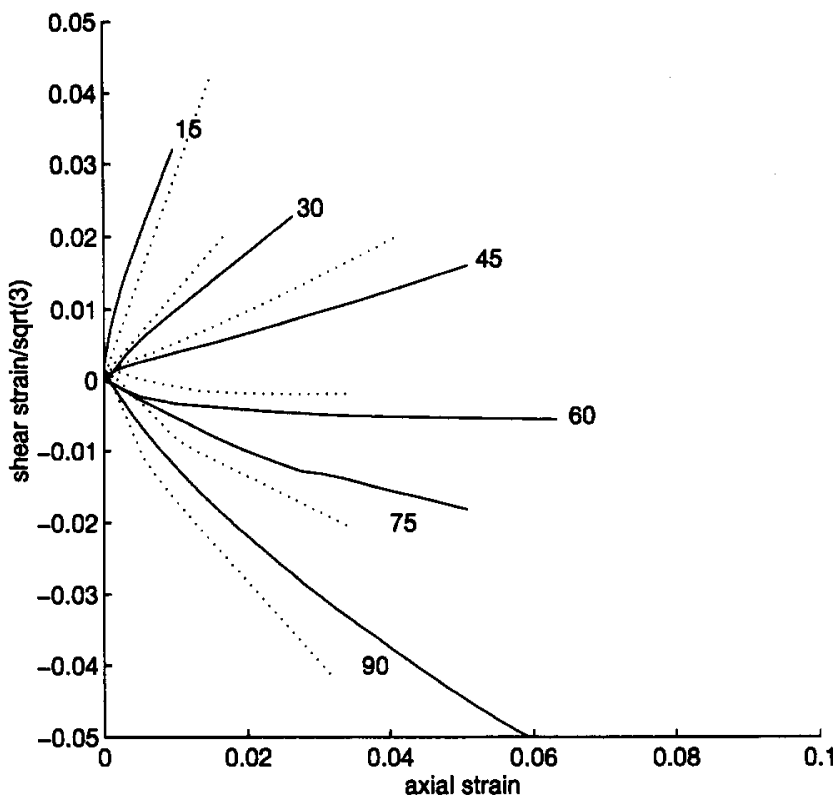

Fig. 4. Comparison of directions of plastic strain rates. _-Experiments; -..... predictions of the model.

plasticity theory of crystals, rather than the yield surface and the commonly used normality rule. Actually, after the direction-dependent quantities, instead of state-dependent quantities, are used to describe the plasticity-induced anisotropic behavior, the conventional normality rule still holds for the direction-dependent quantities in relation to the yield surface. This treatment is an extension of the normality rule to the case of strong anisotropy. To show the effect of this extension, Fig. 5 represents the predictions of other three models given by Khan and Cheng (1996a), in which (a) is the result of Chaboche's (1986) super-positional nonlinear model, (b) is the result of the endochronic model (Valanis, 1971; Valanis and Lee, 1982; Watanabe and Atluri, 1986 and (c) is the result of the two-surface model (Dafalias and Popov, 1976; Dafalais, 1983. Comparison of Fig. 4 with Fig. 5 shows that the prediction of this model does not have as systematic a departure from the experimental data as do those of other models.

Figure 6 shows the experimental data and the prediction of the model concerning the shear stress--strain relation after the first-stage twisting load. The shear strain is the relative deformation to the beginning of the non-proportional loading. The results show that the prediction agrees with the experimental data.

\section{FURTHER DEVELOPMENT OF THE MODEL}

The success of the prediction of the constitutive model to the anisotropic behavior of OHFC copper under non-proportional finite deformations encourages us to consider the further development of the model. Among the potential aspects to be developed, the nonSchmid effect and the visco-plasticity seem significant.

As pointed out previously, the key point of the model lies in the description of the plasticity-induced anisotropic behavior. Instead of using many state-dependent internal 

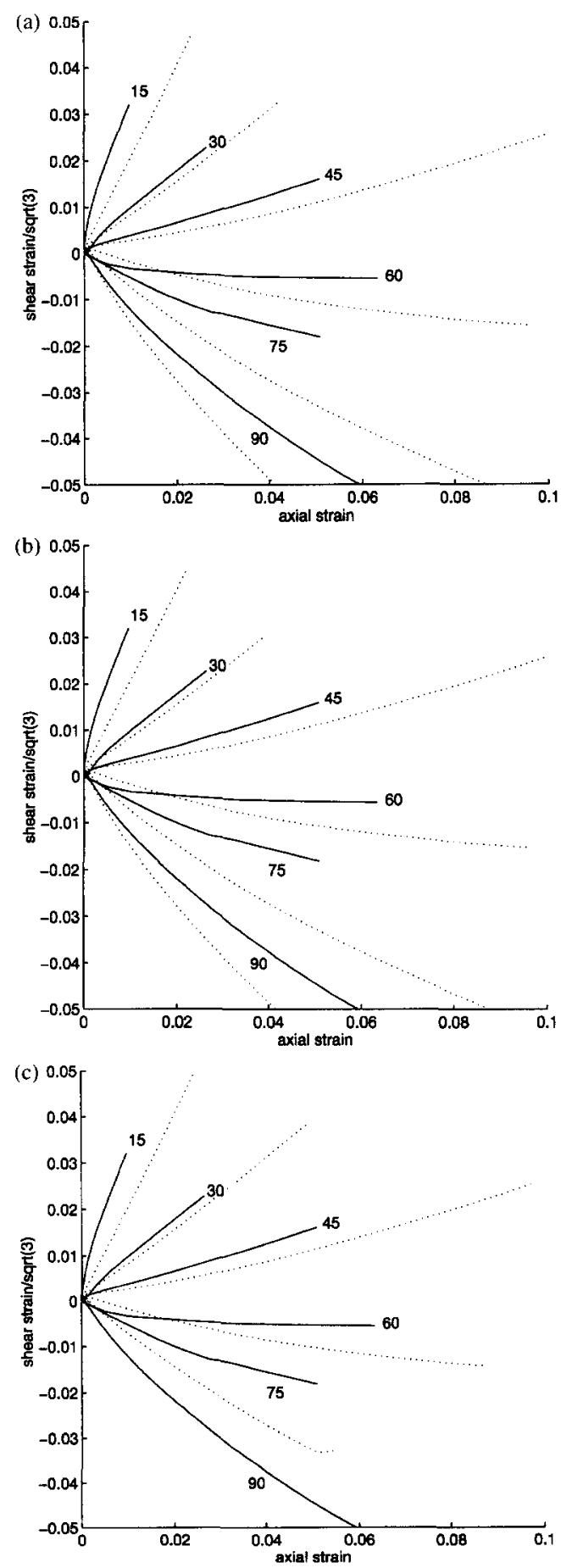

Fig. 5. Comparison of directions of plastic strain rates: (a) _Experiments; ......Chaboche's (1986) model; (b) _experiments; ......Valanis (1971), Valanis and Lee's (1982), Watanabe and Atluri's (1986) model and (c) _experiments; …...Dafalias and Popov's (1976) model. 


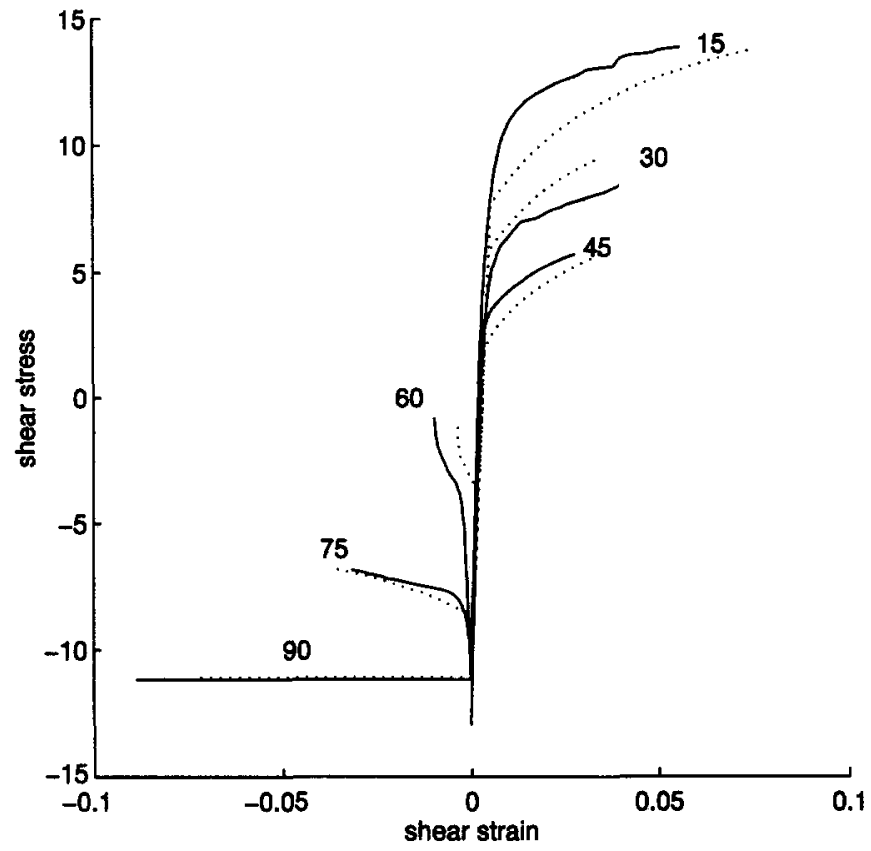

Fig. 6. Comparison of shear stress-strain relations under tension-torsion deformation. - Experiments; .....predictions of the model.

variables as an extension of the conventional Mises-type flow theory, this model is based on direction-dependent variables and focuses on the interaction among them. As a simple and natural extension of the plasticity theory of crystals, the direction-dependent variables are a large number of randomly distributed slip systems. All of the descriptions of slip systems, including the relation between slips and plastic strain rate and the Schmid law, are applied to the direction-dependent variables. As we know, the Schmid law is the same as the Tresca criterion of the maximum shear stress in the case of randomly distributed slip systems. If the Tresca criterion is changed to another criterion, such as Mises-type criterion, as the phenomenological experimental data on the initial yield surface suggested, the Schmid law has to be changed, or the non-Schmid effect needs to be added, or in a general sense, the direction-dependent variables do not necessarily need to be slip systems.

One way to include the non-Schmid effect is to consider the representation of a stress state of a material element. It is known that a stress can be expressed by three principal values. If the volume part of a stress is supposed to be independent of plastic deformation, the plastic behavior can be described with just two of the three values. The maximum shear stress is one of them. Thus, the Schmid law is one parameter approach to the plastic behavior. The non-Schmid effect is the effect of the other parameter. If $\sigma_{1}, \sigma_{2}$ and $\sigma_{3}$ denote the three principal stresses, where $\sigma_{1} \leq \sigma_{2} \leq \sigma_{3},\left(\sigma_{30}-\sigma_{1}\right) / 2$ is the maximum shear stress, then $\sigma_{30}-\sigma_{2}$ may be used to describe the non-Schmid effect. However, mechanism of plastic deformation is mainly due to the shear deformation, and it is better to describe plastic deformation in terms of shear stresses. It is easily verified that a deviatoric stress $s$ can be expressed as two shear stress values. For instance, $\mathbf{s}$ can be written as 


$$
\mathbf{s}=\tau \mathbf{P}+\sqrt{2} \tau \cos \phi \mathbf{P P}+\sqrt{2} \tau \sin \phi \mathbf{P P P}
$$

where

$$
\begin{gathered}
\mathbf{P}=\frac{1}{2}(\mathbf{m} \otimes \mathbf{n}+\mathbf{n} \otimes \mathbf{m}), \\
\mathbf{P P}=\frac{1}{2}(\mathbf{m} \otimes \mathbf{k}+\mathbf{k} \otimes \mathbf{m}), \\
\mathbf{P P P}=\frac{1}{2}(\mathbf{k} \otimes \mathbf{n}+\mathbf{n} \otimes \mathbf{k}),
\end{gathered}
$$

$\mathbf{n}, \mathbf{m}$ and $\mathbf{k}$ are three unit vectors perpendicular one to another, $\tau$ and $\phi$ are two parameters. Obviously, the first term of this expression corresponds to the Schmid law, and the rest may be used to describe the non-Schmid effect. In this case, the direction-dependent variables are represented by $\mathbf{P}+\sqrt{2} \cos \phi \mathbf{P P}+\sqrt{2} \sin \phi \mathbf{P P P}$ with $\mathbf{P}, \mathbf{P P}$ and PPP taking many values in the three-dimensional space. By using the yield criterion that a stress times the direction-dependent variables reaches a critical stress, the Mises yield surface is obtained when every direction has the same critical stress. Moreover, the directiondependent variables are related not only to the geometrical quantities $\mathbf{P}, \mathbf{P P}$ and PPP, but also to the stress parameter $\phi$. The necessary introduction of parameter $\phi$ into the variables can also be explained with the viewpoint of the relation between the variables and plastic strain rate. Normally, each variable corresponds to the direction of a plastic strain rate. As we know, the direction of a plastic strain rate has four independent components under a general stress state, but in three-dimensional space, there are only three independent geometrical variables. It is necessary to include a stress parameter, e.g. $\phi$, to resolve the difference.

This model can also be extended to include the effect of deformation rate. The model includes the description of the behavior of the direction-dependent variables. If every direction is a component of deformation, the effect of the deformation rate could be added to each of the components.

A simple way of considering the effect of deformation rate in this model is to use the concept of over-stress for every direction like the description of one-dimensional viscoplastic stress-strain relations (e.g. Perzyna, 1963; Yao and Krempl, 1985). Let $\tau, \tau_{c}^{i}$ and $\dot{\gamma}^{i}$ denote the stress, the static critical stress and plastic deformation of the $i$ th direction, respectively. Then, the following power function may be a good approximation of the viscoplastic stress-strain relations of every direction:

$$
\dot{\gamma}^{i}=A\left\langle\left|\frac{\tau^{i}}{\tau_{c}^{i}}\right|-1\right\rangle^{n}
$$

where $A$ and $n$ are two material parameters, and $\langle x\rangle=x$ for $x>0$ and $\langle x\rangle=0$ for $x \leq 0$. As for the static critical stress $\tau^{i_{c}}$, it is suggested that the hardening given by eqn (34) is applied.

It is noted that the numerical implementation of the elastic-viscoplastic constitutive model is much simpler than that of the elastic-plastic constitutive model. In the elasticviscoplastic constitutive model, plastic deformation rate, $\dot{\gamma}^{i}$ or $\mathbf{D}^{p}$, is directly calculated 
from stress and hardening which are already known from an incremental calculation process. While in the elastic-plastic constitutive model, plastic deformation depends on stress and hardening rates which are not totally known before the calculation and an iterative calculation is usually required. More significantly, there is no problem concerning the existence of $\left(g^{i j-1}\right.$ in eqn (34) in the elastic-viscoplastic constitutive model. Therefore, the application of the elastic-viscoplastic constitutive model is preferred in practice to that of the elastic-plastic constitutive model. For instance, if $A$ and $n$ in the above equation are all taken to be 1 , the difference between $\tau^{i}$ and $\tau_{c}^{i}$ will be negligible when $\dot{\gamma}^{i}$ is the order of $10^{-4}$ and the elastic-viscoplastic constitutive model will reduce to the elastic-plastic constitutive model.

\section{CONCLUSIONS}

1. The comparison of the prediction of the model to the data concerning stress-strain relations of OHFC copper under the non-proportional finite deformation shows: (a) that the description of polycrystalline metals in terms of a large number of randomly distributed director-slip systems could be a practical anisotropic elastic-plastic constitutive model; (b) that the approach to the interaction among the multiple directors with a three-degree polynomial of their relative locations satisfactorily describes the shape change of the subsequent yield surface with plastic deformation and (c) that the Armstrong and Frederick nonlinear evolution equation of the hardening of every director is a good approximation of the nonlinear stress-strain relations.

2. Most of the material parameters in the model could be easily estimated and determined from experimental data from an one-dimensional stress-strain relation and a subsequent yield surface. The final determination of the material parameters, as usual, should be completed through the numerical implementation of the model.

3. The further development of the model is possible; the generalized directors, instead of slip systems, and the inclusion of the effect of deformation rate are outlined as some examples.

4. The detailed study of the model is needed for other materials or other complicated deformation histories to examine other aspects of the model.

Acknowledgements-The financial support of the National Science Foundation, through grant No. MSS9296045, Mechanics \& Materials Program, is sincerely appreciated.

\section{REFERENCES}

Armstrong, P. J. and Frederick, C. O. (1966) A mathematical representation of the multi-axial Bauschinger effect. G. E. G. B. Report RD/B/N 731 .

Asaro, R. J. (1983) Micro-mechanics of crystals and polycrystals. Advances in Mechanics 23, 1.

Chaboche, J. L. (1986) Time-independent constitutive theories for cyclic plasticity. Int. J. Plasticity 2, 149.

Dafalias, Y. F. (1983) Corotational rates for kinematic hardening at large plastic deformations. ASME J. Appl. Mech. 50, 561.

Dafalias, Y. F. and Popov, E. P. (1976) Plastic internal variables formulatism of cyclic plasticity. ASME J. Appl. Mech. 43, 645 .

Khan, A. S. and Cheng, P. (1996a) Evaluation of three elastic-plastic constitutive models by non-proportional finite deformations of OFHC Copper. Int. J. Plasticity 12, 737. 
Khan, A. S. and Cheng, P. (1996b) An anisotropic elastic-plastic constitutive model for single and polycrystalline metals: I-theoretical development. Int. J. Plasticity 12, 147.

Khan, A. S. and Huang, S. (1995) Continuum Theory of Plasticity. John Wiley and Sons, New York.

Khan, A. S. and Parikh, Y. (1986) Large deformation in polycrystalline copper under combined tension-torsion loading, unloading and reloading or reverse loading: a study of two incremental theories of plasticity. Int. J. Plasticity 2, 379.

Khan, A. S. and Wang, X. (1993) An experimental study on subsequent yield surface after finite shear prestraining. Int. J. Plasticity $9,889$.

Naghdi, P. M., Essenberg, F. and Koff, W. (1958) An experimental study of initial and subsequent yield surfaces in plasticity. J. Appl. Mech. 25, 201.

Perzyna, P. (1963) The constitutive equations for rate sensitive plastic materials. Quarterly Appl. Math. $20,321$.

Phillips, A. and Das, P. K. (1985) Yield surfaces and loading surfaces of aluminum and brass: an experimental investigation at room and elevated temperature. Int. J. Plasticity $1,89$.

Prager, W. (1949) Recent developments in the mathematic theory of plasticity. J. Appl. Phys. $20,235$.

Stout, M. G., Martin, P. L., Helling, D. E. and Canova, G. R. (1985) Multi-axial yield behavior of 1100 aluminum following various magnitudes of prestrain. Int. J. Plasticity $1,163$.

Taylor, G. I. and Quinney, H. (1931) The plastic distortion of metals. Phil. Trans. R. Soc. A230, 323.

Valanis, K. C. (1971) A theory of viscoplasticity without a yield surface, part I: general theory. Arch. Mech. 23, 517.

Valanis, K. C. (1971) A theory of viscoplasticity without a yield surface, part ii: application to the mechanical behavior of metals. Arch. Mech. 23, 535.

Valanis, K. C. and and Lee, C. F. (1982) Some recent developments of the endochronic theory with application. Nuclear Engineering and Design 69, 327.

Watanabe, O. and Atluri, S. N. (1986) Constitutive modelling of cyclic plasticity and creep using an internal time concept. Int. J. Plasticity $2,107$.

Yao, D. and Krempl, E. (1985) Viscoplasticity theory based on over-stress. the Prediction of monotonic and cyclic proportional and non-proportional loading paths of an aluminum alloy. Int. J. Plasticity 1, 259.

Ziegler, H. (1959) A modification of prager's hardening rule. Quart. Appl. Math. 17, 55. 